\title{
Conditional Survival Estimates Improve Over Time for Patients with Hepatocellular Carcinoma: An Analysis for Nationwide Korea Cancer Registry Database
}

\author{
Jae Seung Lee, $\mathrm{MD}^{1,2}$ \\ In Rae Cho, MD ${ }^{1,23}$ \\ Hye Won Lee, MD, PhD',2 \\ Mi Young Jeon, MD1,2 \\ Tae Seop Lim, MD1,2 \\ Oidov Baatarkhuu, MD, PhD ${ }^{1,4}$ \\ Do Young Kim, MD, PhD $D^{1,25}$ \\ Kwang-Hyub Han, MD, PhD ${ }^{1,25}$ \\ Jun Yong Park, MD, PhD $1,2,5$
}

\begin{abstract}
Purpose
Conditional survival estimates (CSE) can provide additional useful prognostic information on the period of survival after diagnosis, which helps in counseling patients with cancer on their individual prognoses. This study aimed to analyze conditional survival (CS) for hepatocellular carcinoma (HCC) using a Korean national registry.
\end{abstract}

\section{Materials and Methods}

Patients with HCC, registered in the Korean cancer registry database, were retrospectively reviewed. Overall survival (OS) was calculated using the Kaplan-Meier method. The 1-year $\mathrm{CS}$ at $\mathrm{X}$ year or month after diagnosis were calculated as $\mathrm{CS}_{1}=\mathrm{OS}(\mathrm{X}+1) / \mathrm{OS}(\mathrm{x})$. CS calculations were performed in each Barcelona Clinic Liver Cancer stage, after which patients at stage $0, A$, and $B$ underwent subgroup analysis using initial treatment methods.

\section{Results}

A total of 4,063 patients diagnosed with HCC from January 2008 to December 2010, and 2,721 who were diagnosed from January 2011 to December 2012, were separately reviewed. In 2008-2010, the 1-year CS of 1, 2, 3, 4, and 5-year survivors was $82.9 \%, 85.1 \%$, $88.3 \%, 88.0 \%$, and $88.6 \%$, respectively. Patients demonstrated an increase in CSE over time in subgroup analysis, especially in the advanced stages. In 2011-2012, the 1-year CS of $6,12,18,24,30$, and 36 months was $81.5 \%, 83.8 \%, 85.3 \%, 85.5 \%, 86.5 \%$, and $88.8 \%$, respectively. The subgroup analysis showed the same tendency towards increased CSE in the advanced stages.

\section{Conclusion}

Overall, the CS improved with each additional year after diagnosis in both groups. CSE may therefore provide a more accurate prognosis and hopeful message to patients who are surviving with or after treatment.

\section{Key words}

Received August 22, 2018

Accepted February 7, 2019

Published Online February 12, 2019

Carcinoma, Hepatocellular, Conditional survival estimate, Survival, Prognosis, Demographic, Republic of Korea

\section{Introduction}

Hepatocellular carcinoma (HCC) is a common type cancer and the second most common cause of cancer-related mortality worldwide [1]; it is a significant health issue in Korea [2]. Although the incidence of HCC has been declining and the 5-year survival rate has been improving recently in Korea, in most patients, HCC is still diagnosed in the advan- ced stage, with extensive tumor burden, vascular invasion, extrahepatic spread, or decompensated liver function; such heterogeneous characteristics can affect treatment decisions and patient survival [3]. In the national randomized registration report for 2003-2005, the 5-year survival rate of Korean HCC patients in the modified Union for International Cancer Control stage I was 52\% and 36\% in stage II, but only $15 \%$ in stage III, sharply decreasing to $6 \%$ in stages IV-A and IV-B each [2]. 
Overall survival (OS), measured from the time of diagnosis, is a traditional and well-established method to predict the long-term prognosis of disease $[4,5]$. However, OS is a relatively static concept and does not reflect the impact of changing variables. For instance, preservation of liver function in patients cured through resection or transarterial therapies is different in each person. The concept of conditional survival estimates (CSE) applies this heterogeneous and changeable patient status and determines the probability that a patient who has survived for a designated period will be alive at another fixed interval [6-8]. CSE is a simple diagram that can predict individual risk over a specified period of time and more accurate prognosis in a group of patients who are at high risk of death at the beginning of the diagnosis [9-12]. Such dynamic prognostic information could help clinicians to provide education, surveillance, management strategies, and life-changing decisions according to individual survival characteristics [13].

Till date, the CSE analyses for HCC were limited to sporadic single-center reports for patients in early stages of HCC who had undergone curative treatment $[14,15]$, or those in advanced stages who had undergone transarterial therapies [16]. The present study aimed to (1) analyze the large cohort of HCC patients that was obtained from the Korea cancer registry database, (2) compare OS and conditional survival (CS) for HCC patients, and (3) identify CSE according to the Barcelona Clinic Liver Cancer (BCLC) staging system, which reflects the patient's performance status, tumor burden, and liver function [17].

\section{Materials and Methods}

\section{Study design}

The Ministry of Health and Welfare, South Korea initiated a nationwide cancer registry in 1980, called the Korea Central Cancer Registry (KCCR). The C22.0 coding system, which is based on the International Classification of Disease 10th edition (ICD-10), was used to abstract HCC patients from the KCCR registry. The National Cancer Center and Korean Liver Cancer Study group studied two periods, from January 2008 to December 2010 and from January 2011 to December 2012, in the KCCR database using the random sample audit method.

A total of 4,597 patients were diagnosed as having HCC

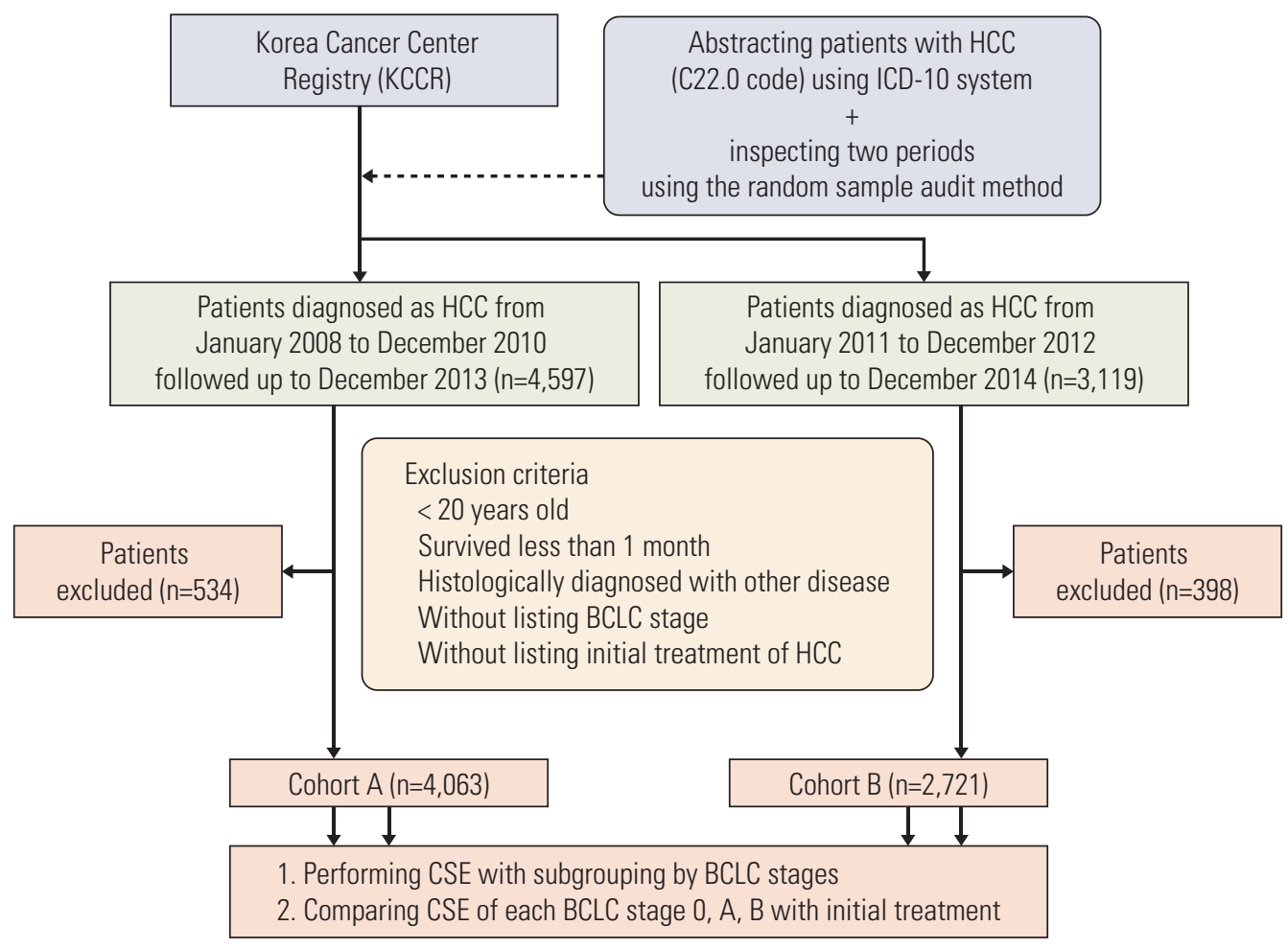

Fig. 1. Flow of selection of the study population. HCC, hepatocellular carcinoma; ICD-10, International Classification of Disease 10th edition; BCLC, Barcelona Clinic Liver Cancer; CSE, conditional survival estimate(s). 
from January 2008 to December 2010 and were followed up until December 2013 in the Korean cancer registry database. We excluded 534 patients who (1) were younger than 20 years, (2) survived less than 1 month from the date of diagnosis, (3) were finally diagnosed with other diseases such as neuroendocrine carcinoma, metastatic carcinoma, cholangiocarcinoma or sarcoma by pathology, (4) did not have the BCLC stage listed, (5) had no record of the initial treatment of HCC, or (6) lacked the important clinical or laboratory parameters explained below. The remaining 4,063 patients were referred to as cohort A. For the period from January 2011 to December 2012 and follow-up to December 2014, 3,119 patients were reviewed. Among these, 398 patients were excluded using the same criteria, and the remaining 2,721 patients were referred to as cohort B. Cohorts A and B were reviewed separately in this study (Fig. 1).

The primary endpoint was performing CSE with secondary subgroup analysis according to the BCLC stages, and secondary endpoint was comparing CSE of each of the BCLC stages $0, A$, and $B$, with subgroup analysis according to initial treatment methods.

\section{Data collection}

Clinical parameters included age, sex, presence of chronic illness such as hypertension or diabetes mellitus, suspected etiologies of HCC such as viral hepatitis or heavy alcohol intake, ascites, hepatic encephalopathy (HEP), number and maximum size of tumors, presence of portal vein invasion and metastasis to regional lymph node or distant organ, Child-Pugh score and classification, model for end-stage liver disease (MELD) score, BCLC stages at the time of initial diagnosis, and initial treatment methods. Ascites was re-classified into "controlled" and "uncontrolled" (grade 2-3) [18], and HEP was re-classified into "mild" (grade 1-2) and "severe" (grade 3-4) according to the West-Haven criteria [19]. Laboratory parameters included platelet level, serum sodium, albumin, total bilirubin, creatinine, alanine aminotransferase, international normalized ratio (INR), and $\alpha$-fetoprotein. The Child-Pugh score was assessed according to the standard criteria [20]. MELD score were calculated according to the Malinchoc formula: $\mathrm{R}=9.57 \times \ln ($ creatinine $[\mathrm{mg} / \mathrm{dL}])+$ $3.78 \times \ln ($ bilirubin $[\mathrm{mg} / \mathrm{dL}])+11.2 \times \ln (\mathrm{INR})+6.43 \times($ etiology: 0 if cholestatic or alcoholic, 1 otherwise) [21]. Other clinical or laboratory parameters were excluded due to insufficient medical record.

\section{Treatment methods}

Patients diagnosed with HCC underwent various initial treatments, including surgical resection, liver transplantation (LT), local ablation, transarterial therapy, systemic chemo- therapy (CTx), and radiation therapy (RTx). Local ablation therapy included radiofrequency ablation (RFA), alcohol injection, and other local ablation such as cryoablation. Transarterial therapy included transarterial chemoembolization (TACE) with gelatin sponge (gelform), transarterial chemolipiodolizaiton (without gelform), TACE with beads, yttrium90 radioembolization, and transarterial chemoinfusion via catheter or intraarterial chemoport. Systemic CTx included sorafenib and other systemic CTx. Some patients did not undergo any treatments.

\section{Statistical analysis}

Student $t$ test, Mann-Whitney test, chi-square test, or Kappa statistics were used for comparing variables and calculating $\mathrm{p}$-values as appropriate. The OS was computed from the day treatment began until the most recent follow-up or death. Survival time and rate were estimated using the KaplanMeier method, and differences between groups were assessed using the log-rank test. Cox proportional hazard ratios were calculated to test the association between the clinical parameters and OS. All variables found to be significant in the univariate analysis were included in the Cox multivariate analysis. Statistical analyses were conducted using the IBM SPSS Statistics software, ver. 23.0.0.0 (IBM Corp., Armonk, NY), and a two-sided $\mathrm{p}$-value of $<0.05$ was considered significant.

CS estimates can be calculated from traditional KaplanMeier and actuarial life table survival data. The mathematical definition of CS is expressed as follows: $\mathrm{CS}(\mathrm{y} \mid \mathrm{x})$ is the probability of surviving for an additional y years or months, given that the patient has already survived $x$ years or months. If $S(t)$ is assumed to be the traditional actuarial survival at time $t$; $\mathrm{CS}$ can be calculated as: $\mathrm{CS}(\mathrm{y} \mid \mathrm{x})=\mathrm{S}(\mathrm{x}+\mathrm{y}) / \mathrm{S}(\mathrm{x})$. For example, to determine the 1-year CSE for a patient who has already survived 2 years, we simply divide the survival estimate at $\mathrm{t}=1+2$ by the survival at $\mathrm{t}=2: \mathrm{CS}(1 \mid 2)=S(5) / \mathrm{S}(2)$.

Cumulative CS calculations were performed with secondary subgroup analyses using BCLC stages. Then, additional subgroup analyses were performed for the patients in stages 0 , A, and B using the initial treatment methods.

\section{Ethical statement}

This study was performed in accordance with the ethical guidelines of the 1975 Declaration of Helsinki and was approved by the institutional review board of Yonsei University Health System (IRB No. 4-2014-1088). The need for written informed consent was waived because of the retrospective nature. 
Table 1. Baseline characteristics of patients in cohort A (diagnosed in 2008-2010) and cohort B (diagnosed in 2011-2012)

\begin{tabular}{|c|c|c|c|}
\hline Variable & Cohort A $(n=4,063)$ & Cohort $B(n=2,721)$ & p-value $e^{a}$ \\
\hline Age (yr) & $59.0(51.0-68.0)$ & $60.0(52.0-69.0)$ & $<0.001$ \\
\hline Male sex & $3,144(77.4)$ & 2,202 (80.9) & $<0.001$ \\
\hline Body mass index $\left(\mathrm{kg} / \mathrm{m}^{2}\right)$ & $23.7(21.7-25.8)$ & $23.6(21.6-25.9)$ & 0.824 \\
\hline Hypertension & $1,218(30.0)$ & 887 (32.6) & 0.027 \\
\hline Diabetes mellitus & $937(23.1)$ & $632(23.2)$ & 0.910 \\
\hline Viral etiology & $2,962(72.9)$ & $1,990(73.1)$ & 0.326 \\
\hline Heavy alcohol intake & $1,231(30.3)$ & $842(30.9)$ & 0.876 \\
\hline Ascites & $889(21.9)$ & $591(21.7)$ & 0.968 \\
\hline Hepatic encephalopathy & $89(2.2)$ & $57(2.1)$ & 0.675 \\
\hline Multiple tumors & $1,528(37.6)$ & $1,009(37.1)$ & 0.686 \\
\hline Above triple tumors & $942(23.2)$ & $618(22.7)$ & 0.668 \\
\hline Maximum tumor diameter $(\mathrm{cm})$ & & & 0.858 \\
\hline$>3$ & 2,217 (54.6) & $1,468(54.0)$ & 0.595 \\
\hline Portal vein invasion & $905(22.3)$ & $554(20.4)$ & 0.060 \\
\hline Regional LN metastasis & $263(6.5)$ & $167(6.1)$ & 0.578 \\
\hline Distant metastasis & $350(8.6)$ & $236(8.7)$ & 0.932 \\
\hline Platelet $\left(\times 10^{9} / \mathrm{L}\right)$ & $136(92-197)$ & $141(96-194)$ & 0.430 \\
\hline Serum sodium $(\mathrm{mmol} / \mathrm{L})$ & $139(137-141)$ & $139(137-141)$ & 0.173 \\
\hline ALT (IU/L) & $37.0(24.0-59.0)$ & $35.0(23.0-55.0)$ & $<0.001$ \\
\hline Albumin $(g / L)$ & $3.8(3.3-4.2)$ & $3.9(3.3-4.2)$ & $<0.001$ \\
\hline Total bilirubin (IU/L) & $0.99(0.70-1.50)$ & $0.90(0.62-1.40)$ & 0.001 \\
\hline Serum creatinine $(\mathrm{IU} / \mathrm{L})$ & $0.90(0.75-1.00)$ & $0.87(0.72-1.00)$ & 0.101 \\
\hline Prothrombin time (INR) & $1.11(1.04-1.22)$ & $1.10(1.03-1.19)$ & $<0.001$ \\
\hline$\alpha$-Fetoprotein $(\mathrm{ng} / \mathrm{mL})$ & $41.3(6.9-625.8)$ & $29.6(6.3-483.0)$ & 0.680 \\
\hline Child-Pugh class & & & 0.076 \\
\hline B (7-9) & $912(22.4)$ & $569(20.9)$ & - \\
\hline C (10-15) & $156(3.8)$ & $88(3.2)$ & - \\
\hline MELD & $9.00(7.00-11.00)$ & $8.00(7.00-10.00)$ & $<0.001$ \\
\hline BCLC stage & & & 0.006 \\
\hline Stage A & $1,730(42.6)$ & $1,118(41.1)$ & - \\
\hline Stage B & $441(10.9)$ & $319(11.7)$ & - \\
\hline Stage C & $1,348(33.2)$ & $870(32.0)$ & - \\
\hline Stage D & $202(5.0)$ & $118(4.3)$ & - \\
\hline
\end{tabular}

Variables are presented as median (interquartile range) or number (\%). LN, lymph node; ALT, aminotransferase; INR, international normalized ratio; MELD, model for end-stage liver disease; BCLC, Barcelona-clinic liver cancer. ${ }^{a}$ Student $\mathrm{t}$ test, Mann-Whitney test, chi-square test, or Kappa statistics were used for calculating p-values as appropriate.

\section{Results}

\section{Patient characteristics}

A total of 4,063 patients in cohort A and 2,721 in cohort B were separately reviewed. The demographics and clinical characteristics of each group are summarized in Table 1 and S1 Table.

In Cohort $\mathrm{A}$, during the median follow-up of 33.0 months (interquartile range [IQR], 7.3 to 50.5), 2,436 patients died, with a median OS of 10.5 months (IQR, 3.7 to 25.8). The median age was 59.0 years (IQR, 51.0 to 68.0), higher with the mortality (60.0 years; IQR, 52.0 to $69.0 ; \mathrm{p}<0.001$ ). Male sex was predominant $(77.4 \%)$, and chronic hepatitis B was more frequent than other etiologies $(60.7 \%)$. Patients with mortality tended to show further progression in the BCLC staging system. In cohort B, the median follow-up period was 27.8 months (IQR, 9.7 to 36.6) shorter than that in cohort A. The median OS of 1,294 patients with mortality was 8.8 months (IQR, 3.6 to 19.8). Several parameters showed the same tendency as those seen in cohort A. Although some 


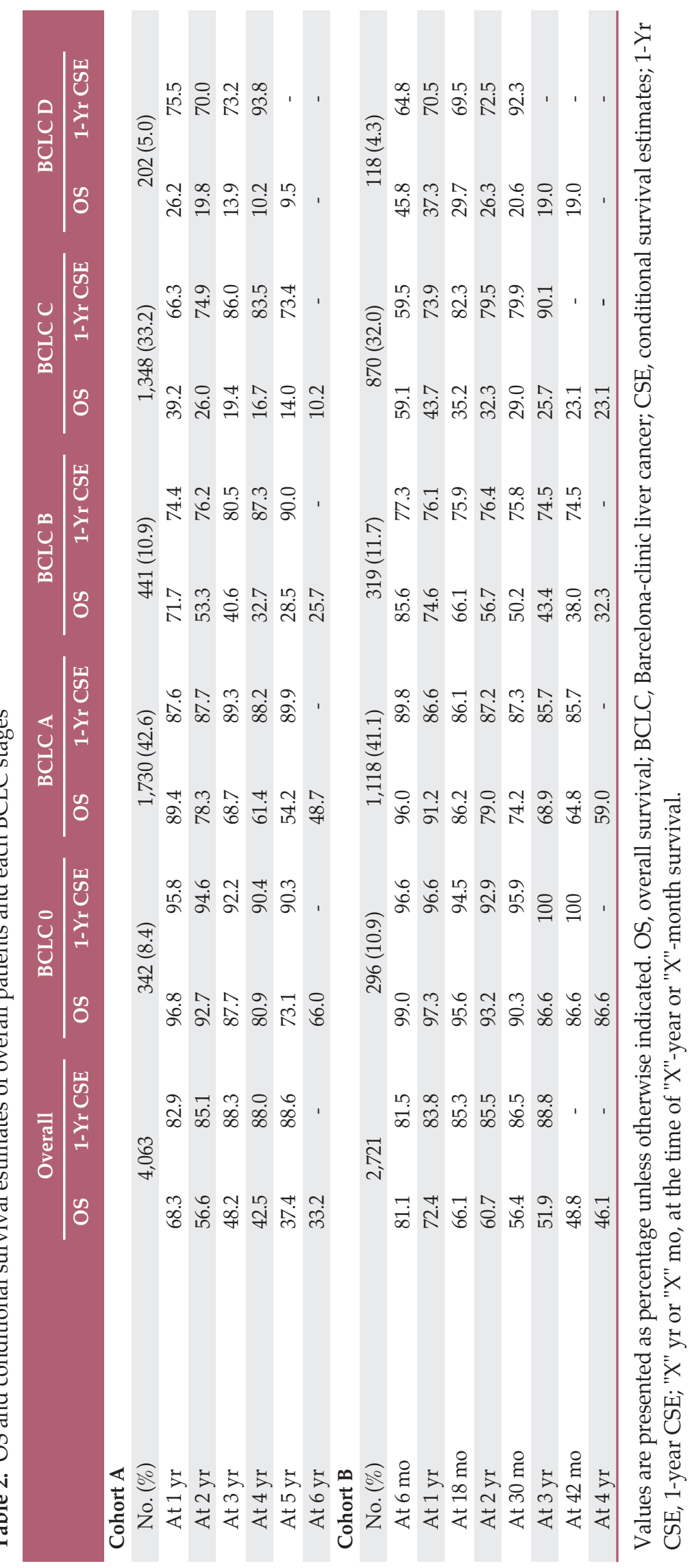


A
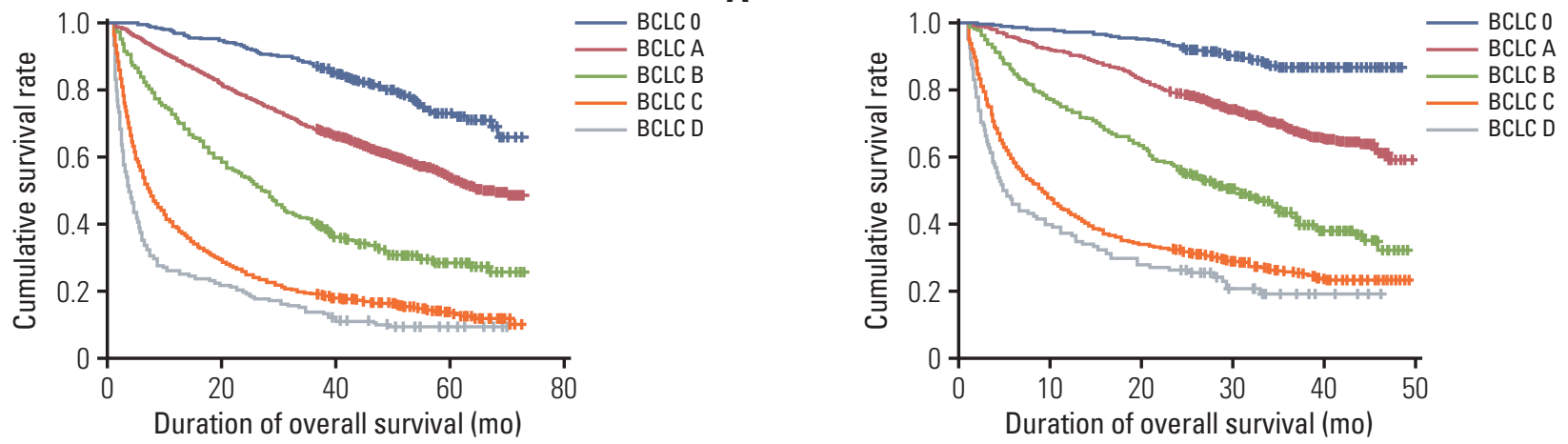

Fig. 2. Kaplan-Meier curves for cumulative survival rate of each Barcelona Clinic Liver Cancer (BCLC) stage in cohort A diagnosed in 2008-2010 (A) and cohort B diagnosed in 2011-2012 (B). Log-rank tests between any groups; $\mathrm{p}<0.001$.

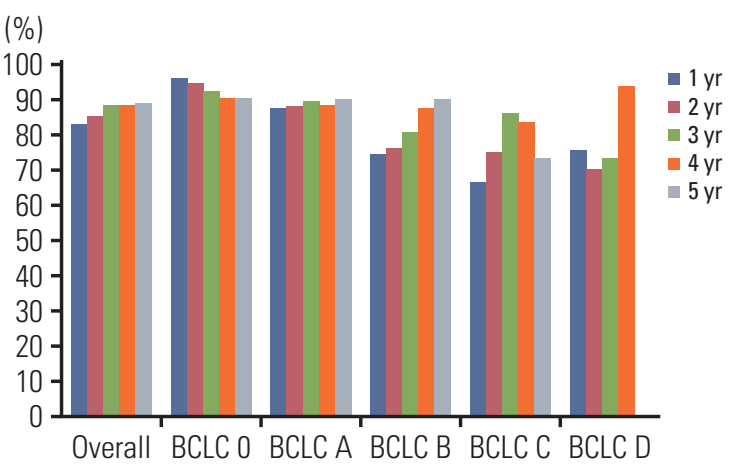

A

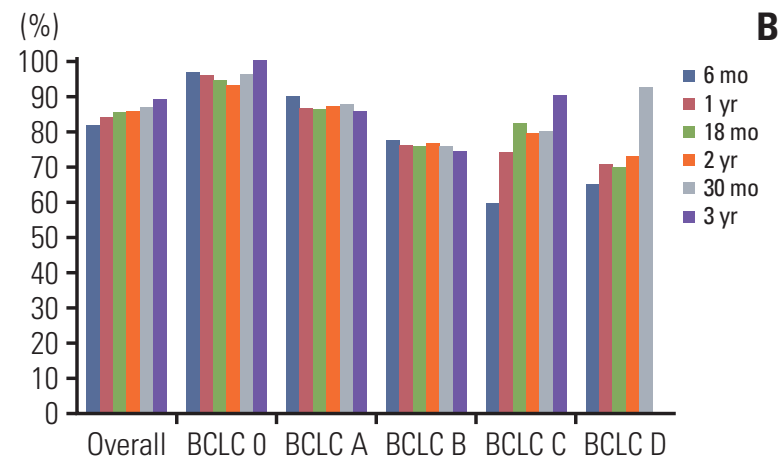

Fig. 3. One-year conditional survival estimates of overall patients and each Barcelona Clinic Liver Cancer (BCLC) stage in cohort A diagnosed in 2008-2010 (A) and cohort B diagnosed in 2011-2012 (B). "X" yr or "X" mo, 1-year conditional survival estimates at the time of "X"-year or "X"-month survival.

parameters showed significant differences between two cohorts, the mean or median of the parameters was almost similar.

\section{Independent predictor of mortality}

In univariate analysis, almost all variables were significantly associated with mortality (S2 Table), except the variables such as hypertension and diabetes mellitus. In the multivariate analysis (S3 Table), some variables at baseline were significantly associated with mortality in both cohorts: age, body mass index, ascites, multiple tumors, maximum tumor diameter above $3 \mathrm{~cm}$, portal vein invasion, distant metastasis, serum albumin, prothrombin time, and MELD score. However, the multivariate analysis contained many missing values, $987(24.3 \%)$ in cohort A and $476(12.5 \%)$ in cohort $\mathrm{B}$, respectively.

\section{OS and CSE for overall patients}

The cumulative survival rates of each BCLC stage, calculated using Kaplan-Meier analysis, are summarized in Table 2 and Fig. 2. Because of the relatively short follow-up period of the cohorts, we calculated only 1-year CSE, at 1-year intervals in cohort $\mathrm{A}$ and 6-month intervals in cohort $\mathrm{B}$.

In cohort A, the OS for overall patients at the time of 1-, 2-, $3-, 4-, 5-$, and 6-year survival were $68.3 \%, 56.6 \%, 48.2 \%$, $42.5 \%, 37.4 \%$, and $33.2 \%$, respectively. The 1-year CSE for 1-, $2-, 3-, 4-$, and 5-year survivors was $82.9 \%, 85.1 \%, 88.3 \%$, $88.0 \%$, and $88.6 \%$, respectively; as is noted, the CSE tended to increase over time (Table 2, Fig. 3A). In cohort B, the overall OS for 1, 2, 3, and 4 years were $72.4 \%, 60.7 \%, 51.9 \%$, and $46.1 \%$, respectively. The 1-year CSE for 6, 12, 18, 24, 30, and 36 months were $81.5 \%, 83.8 \%, 85.3 \%, 85.5 \%, 86.5 \%$, and $88.8 \%$; this index also showed a tendency increase (Table 2, Fig. 3B). 


\section{CSE analyses for each BCLC stage}

The OS and 1-year CSE of each BCLC stage were provided in Table 2 and Fig. 3. Because of a relatively high OS for the initial 2 years of BCLC stage 0 and A, the initial CSE of the early stages tended to nearly plateau or even slightly decreased over time in cohort A. The mortality rates of BCLC stage $\mathrm{C}$ and D were relatively high. The CSE of the advanced stages tended to increase over time, except a decrease or blank at the time of 4- and 5-year survival, which was suspected to be caused by the high rate of mortality or failure to follow-up (Table 2, Fig. 3A). Cohort B also showed nearly same tendency in CSE as did cohort A, plateauing in the early stages and increasing over time in the advanced stages (Table 2, Fig. 3B). The CSE of BCLC stage B was different between the cohorts, tending to increase in cohort $\mathrm{A}$, but decreasing slightly in cohort B.

\section{CSE analyses for each subgroup of BCLC stage using ini- tial treatment methods}

The survival analyses of the subgroups using initial treatment methods were performed in each BCLC stage. Fig. 4 and S4 Table demonstrate the OS and CSE of the patients in cohort A treated with different methods in BCLC stages 0 , $\mathrm{A}$, and $\mathrm{B}$. Increasing tendencies of CSE were shown in patients who underwent surgical therapy in the early stages and transarterial therapies in the intermediate stage (Fig. 4). In Cohort B (Fig. 5, S5 Table), the increasing tendency was not definitely shown in each subgroup. However, increasing CSE at the time of the last 3-year survival is seen in patients who underwent surgery and local ablation in the early stages and transarterial therapies in the intermediate stage.

\section{CSE analyses for each initial treatment modality and cause of HCC}

The OS and 1-year CSE of each initial treatment modality were provided in S6 Table. Only the patients $(\mathrm{n}=1,959,48.2 \%)$ who treated using transarterial methods showed the increasing tendency of 1-year CSE.

For etiologies of HCC, chronic hepatitis B, hepatitis $\mathrm{C}$ and heavy alcohol intake, the CSE analyses were summarized as S7 Table. For patients with hepatitis B virus, the absolute value of CSE was higher than patients without hepatitis B virus; however, the increase in CSE was not significantly different in both cohorts. The result for heavy alcoholics was inversely related to the result for chronic hepatitis B. Patients with heavy alcohol intake showed consistently low 1-year CSE during the follow-up periods in the two cohorts. About chronic hepatitis $C$, there were no any significant findings.


Fig. 4. One-year conditional survival estimates of each Barcelona Clinic Liver Cancer (BCLC) subgroup using initial treatment methods in cohort $\mathrm{A}$, in BCLC 0 (A), BCLC A (B), and BCLC B (C). "X" yr, 1-year conditional survival estimates at the time of "X"-year survival.

\section{Discussion}

In the present study, both cohorts showed a tendency to increase in the CSE for overall HCC patients, and a greater increase in the CSE in the advanced stages, in spite of the 
(\%)

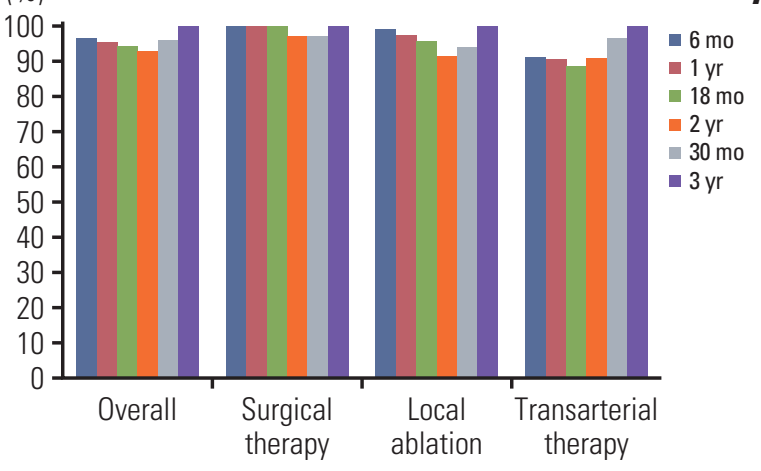

$(\%)$
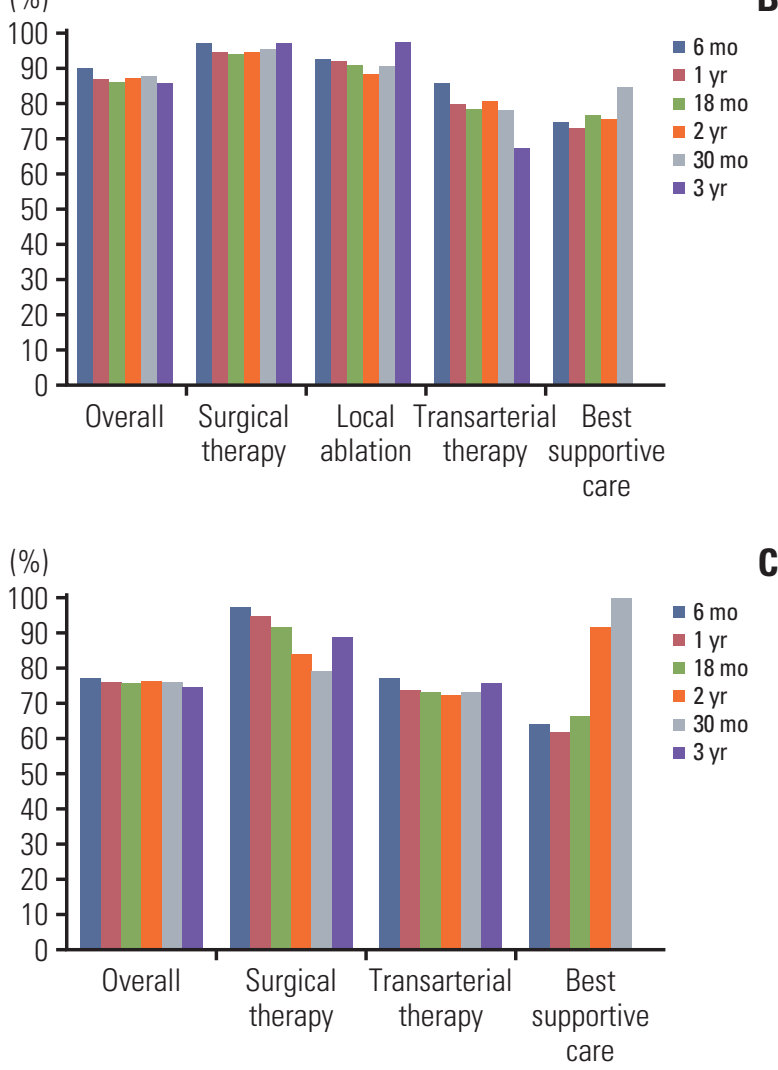

Fig. 5. One-year conditional survival estimates of each Barcelona Clinic Liver Cancer (BCLC) subgroup using initial treatment methods in cohort $\mathrm{B}$, in BCLC 0 (A), BCLC A (B), and BCLC B (C). "X" yr or "X" mo, 1-year conditional survival estimates at the time of "X"-year or "X"-month survival.

high initial mortality. In contrast, CS was minimally changed or even decreased in the early stages. The excess mortality seen in the early stage was minimal during the follow-up period; therefore, the CSE in the early stages would have been significantly affected by less mortality. The CSE in advanced stages were similar at the same time compared with those in early stages, even though the OS was shorter. Thus, when the initial treatment response of patients with HCC in the advanced stage is good, the prognosis can be expected to be similar to that in the early stages. This overall pattern of the stage-specific CSE was similar to that seen in population-based studies of several other cancers [22-24].

We classified patients with initial treatment method after classification with the BCLC stage, because the BCLC stage is determined according to the characteristics of each patients and tumor heterogeneity at the time of diagnosis and provides the recommended initial therapy based on the stage [18]. In general, patients with BCLC stage $0, A$, and B undergo treatment with curative aims, such as surgical resection, LT, local ablation, localized transarterial therapy, or radiation. In the subgroup analysis using the initial treatment methods in each BCLC stage, the CSE tended to increase when the recommended therapy was performed initially, suggesting the effectiveness of these treatment methods. However, this analysis may not be completely accurate, since the long-term analyses or analyses of the cases of multiple or concurrent treatment were lacking in this study. The CSE analyses for each initial treatment modality alone were also done additionally; however, the results may not be very significant because the treatment modality could be affected by the patients' disease status.

In BCLC stage B, the two cohorts showed difference tendencies in CSE. The intermediate and advanced stages include a much wider spectrum of disease and patient performance. This increases the range of possible therapies due to different treatment options, aggressiveness of treatment, patient tolerance, and varying goals of treatment $[25,26]$. These can be considered as a limitation of the BCLC staging system, with difficulty in reflecting the dynamic changes in the variables; this might have caused the heterogeneity of CSE analysis seen in this study. Many experts suggested further sub-classification of the intermediate stage to overcome this heterogeneity and provide proper management to individuals [27-30]. However, the effect of dynamic change of the individual variables is too difficult to measure and predict with the initial staging protocols.

Cucchetti et al. [15] investigated CS for cirrhotic patients in Italy after hepatic resection for HCC. They presented 5-year CS on various baseline characteristics, but the impact of these baseline variables influencing the survival is not linear over time after hepatic resection. The influence of conventional tumor features such as tumor stage, differentiation degree, and vascular invasion was seen only for the first 2 years. Especially, patients with preserved liver function could undergo secondary hepatectomy for recurrent tumor, and CS for these performed secondary surgery tended to increase.

Facciorusso et al. [14] also analyzed long term CS for HCC 
patients performed RFA. In this study, the 5-year CS was decreased significantly after the third-year follow-up. Because RFA was often used as an alternative method in patients unable to undergo liver resection, the influence of the evolutionary liver function and the clinical parameters of each patient would more significantly affect the long-term prognosis. These studies suggested that the dynamic changes in the clinical course should be considered when predicting the prognosis of patients with HCC, and the concept of CSE would provide a useful mechanism to prevent static timeof-diagnosis risk assessment as a fixed predictive moment.

Another 2-year CS analysis in BCLC B and C using hepatic arterial infusion chemotherapy (HAIC) with concurrent RTx (CRT) was carried out in a single tertiary center [16]. In this study, underlying liver function was seen to have a constant effect on CS over time. The analysis showed increasing OS and CS over time in the intermediate and advanced stages; thus, they concluded that HAIC with CRT could be a hopeful option for patients, even in advanced stages. Similarly, CS may enable clinicians to evaluate the consistency of the prognosis to more accurately determine the specific therapy required.

Clinicians may, for example, use CS data in an effort to institute data-driven optimization of post-therapy surveillance. For instance, many physicians minimize follow-up surveillance after 3-5 years, often with little justification based on survival data. Additionally, CS has utility as another mechanism to detect differentials in population survival patterns. The present study can provide a database based on large-scale research, meaning that it can be used to predict the future prognosis based on the information that a particular patient has at a certain point in time. It is important that the rise of CSEs ensures that subsequent prognosis can be better if the survival of patients to a certain point in time is successful. Additionally, CS may force the physician to remember that the risk of death changes as a patient survives for more time from the diagnosis.

There are several limitations in the present study. First, despite enrolling a large number of patients from a national database, there were insufficient or lacking clinical data for evaluation of other variables such as performance status because of retrospective design; therefore, the application of other advanced sub-staging systems or additional CSE according to the concurrent therapies were not available. Second, the follow-up duration was not long enough to confirm the long-term data regarding CS tendency. A larger cohort or prospective survival data with sufficient follow-up would lead to a more accurate CSE, and may provide more helpful clinical information. Third, this study did not analyze changes in patient quality of life, socio-economic status, or disease characteristics and how these affect survival.

In conclusion, the prognosis for overall HCC survivors improves with each additional year or month after initial diagnosis. Quantitative insight into conditional survival may be useful for clinicians to help plan optimal cancer surveillance. Patients who are at more advanced stages in particular, can feel encouraged by their improving prognosis with every year survived. CS can be a useful tool to predict survival for HCC patients and provide them with more relevant and meaningful information concerning the change in their prognosis over time.

\section{Electronic Supplementary Material}

Supplementary materials are available at Cancer Research and Treatment website (https: // www.e-crt.org).

\section{Conflicts of Interest}

Conflict of interest relevant to this article was not reported.

\section{Acknowledgments}

The database used in this study was provided by the Korean Central Cancer Registry, Ministry of Health and Welfare, South Korea, and the Korean Liver Cancer Study Group. This work was supported by a grant from the Korea Health Technology R\&D Project through the Korea Health Industry Development Institute (KHIDI), funded by the Ministry of Health \& Welfare, Republic of Korea (HI15C2859) and the National Research Foundation of Korea (NRF) grant funded by the Korea government (MSIT) (NRF-2018R1A2B2005901).

\section{References}

1. McGlynn KA, Petrick JL, London WT. Global epidemiology of hepatocellular carcinoma: an emphasis on demographic and regional variability. Clin Liver Dis. 2015;19:223-38.

2. Kim BH, Park JW. Epidemiology of liver cancer in South Korea. Clin Mol Hepatol. 2018;24:1-9.
3. Yu SJ. A concise review of updated guidelines regarding the management of hepatocellular carcinoma around the world: 2010-2016. Clin Mol Hepatol. 2016;22:7-17.

4. Terzi E, Golfieri R, Piscaglia F, Galassi M, Dazzi A, Leoni S, et al. Response rate and clinical outcome of HCC after first and 
repeated cTACE performed "on demand". J Hepatol. 2012;57: 1258-67.

5. Jung ES, Kim JH, Yoon EL, Lee HJ, Lee SJ, Suh SJ, et al. Comparison of the methods for tumor response assessment in patients with hepatocellular carcinoma undergoing transarterial chemoembolization. J Hepatol. 2013;58:1181-7.

6. Henson DE, Ries LA. On the estimation of survival. Semin Surg Oncol. 1994;10:2-6.

7. Hieke S, Kleber M, Konig C, Engelhardt M, Schumacher M. Conditional survival: a useful concept to provide information on how prognosis evolves over time. Clin Cancer Res. 2015;21: 1530-6.

8. Zheng MH, Wu SJ, Shi KQ, Yan HD, Li H, Zhu GQ, et al. Conditional survival estimate of acute-on-chronic hepatitis B liver failure: a dynamic prediction based on a multicenter cohort. Oncotarget. 2015;6:23261-71.

9. Bischof DA, Kim Y, Dodson R, Jimenez MC, Behman R, Cocieru A, et al. Conditional disease-free survival after surgical resection of gastrointestinal stromal tumors: a multi-institutional analysis of 502 patients. JAMA Surg. 2015;150:299-306.

10. Kim Y, Ejaz A, Spolverato G, Squires MH, Poultsides G, Fields $\mathrm{RC}$, et al. Conditional survival after surgical resection of gastric cancer: a multi-institutional analysis of the us gastric cancer collaborative. Ann Surg Oncol. 2015;22:557-64.

11. Sun M, Trinh QD, Karakiewicz PI. Conditional survival of patients with metastatic renal-cell carcinoma. Lancet Oncol. 2012;13:e462.

12. Zabor EC, Gonen M, Chapman PB, Panageas KS. Dynamic prognostication using conditional survival estimates. Cancer. 2013;119:3589-92.

13. Ferrell BR, Dow KH, Leigh S, Ly J, Gulasekaram P. Quality of life in long-term cancer survivors. Oncol Nurs Forum. 1995;22: 915-22.

14. Facciorusso A, Del Prete V, Antonino M, Neve V, Amoruso A, Crucinio N, et al. Conditional survival analysis of hepatocellular carcinoma patients treated with radiofrequency ablation. Hepatol Res. 2015;45:E62-72.

15. Cucchetti A, Piscaglia F, Cescon M, Ercolani G, Terzi E, Bolondi L, et al. Conditional survival after hepatic resection for hepatocellular carcinoma in cirrhotic patients. Clin Cancer Res. 2012;18:4397-405.

16. Cho IR, Lee HW, Song KJ, Kim BK, Kim SU, Kim DY, et al. Conditional survival estimate in patients with Barcelona Clinic Liver Cancer stage B / C hepatocellular carcinoma treated with hepatic arterial infusion chemotherapy with/without concurrent radiotherapy. Oncotarget. 2017;8:79914-26.

17. Kim BK, Kim SU, Park JY, Kim DY, Ahn SH, Park MS, et al. Applicability of BCLC stage for prognostic stratification in comparison with other staging systems: single centre experience from long-term clinical outcomes of 1717 treatment-naive patients with hepatocellular carcinoma. Liver Int. 2012;32: 1120-7.

18. European Association for the Study of the Liver. EASL clinical practice guidelines on the management of ascites, spontaneous bacterial peritonitis, and hepatorenal syndrome in cirrhosis. J Hepatol. 2010;53:397-417.

19. Ferenci P, Lockwood A, Mullen K, Tarter R, Weissenborn K, Blei AT. Hepatic encephalopathy-definition, nomenclature, diagnosis, and quantification: final report of the working party at the 11th World Congresses of Gastroenterology, Vienna, 1998. Hepatology. 2002;35:716-21.

20. Pugh RN, Murray-Lyon IM, Dawson JL, Pietroni MC, Williams R. Transection of the oesophagus for bleeding oesophageal varices. Br J Surg. 1973;60:646-9.

21. Malinchoc M, Kamath PS, Gordon FD, Peine CJ, Rank J, ter Borg PC. A model to predict poor survival in patients undergoing transjugular intrahepatic portosystemic shunts. Hepatology. 2000;31:864-71.

22. Yu XQ, Baade PD, O'Connell DL. Conditional survival of cancer patients: an Australian perspective. BMC Cancer. 2012;12: 460.

23. Janssen-Heijnen ML, Gondos A, Bray F, Hakulinen T, Brewster $\mathrm{DH}$, Brenner $\mathrm{H}$, et al. Clinical relevance of conditional survival of cancer patients in europe: age-specific analyses of 13 cancers. J Clin Oncol. 2010;28:2520-8.

24. Anderson C, Smitherman AB, Nichols HB. Conditional relative survival among long-term survivors of adolescent and young adult cancers. Cancer. 2018;124:3037-43.

25. Chow PK, Choo SP, Ng DC, Lo RH, Wang ML, Toh HC, et al. National Cancer Centre Singapore consensus guidelines for hepatocellular carcinoma. Liver Cancer. 2016;5:97-106.

26. Kitai S, Kudo M, Nishida N, Izumi N, Sakamoto M, Matsuyama $Y$, et al. Survival benefit of locoregional treatment for hepatocellular carcinoma with advanced liver cirrhosis. Liver Cancer. 2016;5:175-89.

27. Kudo M. Heterogeneity and subclassification of Barcelona Clinic Liver Cancer Stage B. Liver Cancer. 2016;5:91-6.

28. Yamakado K, Miyayama S, Hirota S, Mizunuma K, Nakamura $\mathrm{K}$, Inaba $\mathrm{Y}$, et al. Prognosis of patients with intermediate-stage hepatocellular carcinomas based on the Child-Pugh score: subclassifying the intermediate stage (Barcelona Clinic Liver Cancer stage B). Jpn J Radiol. 2014;32:644-9.

29. Kudo M, Arizumi T, Ueshima K, Sakurai T, Kitano M, Nishida N. Subclassification of BCLC B stage hepatocellular carcinoma and treatment strategies: proposal of modified Bolondi's subclassification (Kinki Criteria). Dig Dis. 2015;33:751-8.

30. Yau T, Tang VY, Yao TJ, Fan ST, Lo CM, Poon RT. Development of Hong Kong Liver Cancer staging system with treatment stratification for patients with hepatocellular carcinoma. Gastroenterology. 2014;146:1691-700.e3. 